Article

\title{
$S$-Subgradient Projection Methods with S-Subdifferential Functions for Nonconvex Split Feasibility Problems
}

\author{
Jinzuo Chen ${ }^{1}\left(\mathbb{D}\right.$, Mihai Postolache ${ }^{2,3,4, *(\mathbb{D})}$ and Yonghong Yao ${ }^{5,6}$ (i) \\ 1 School of Mathematics and Statistics, Lingnan Normal University, Zhanjiang 524048, China; \\ chanjanegeoger@hotmail.com \\ 2 Center for General Education, China Medical University, Taichung 40402, Taiwan \\ 3 Department of Mathematics and Informatics, University "Politehnica" of Bucharest, \\ 060042 Bucharest, Romania \\ 4 Romanian Academy, Gh. Mihoc-C. Iacob Institute of Mathematical Statistics and Applied Mathematics, \\ 050711 Bucharest, Romania \\ 5 School of Mathematical Sciences, Tianjin Polytechnic University, Tianjin 300387, China; \\ yyhtgu@hotmail.com or yaoyonghong@aliyun.com \\ 6 The Key Laboratory of Intelligent Information and Big Data Processing of NingXia Province, \\ North Minzu University, Yinchuan 750021, China \\ * Correspondence: mihai@mathem.pub.ro
}

Received: 24 November 2019; Accepted: 11 December 2019; Published: 14 December 2019

check for updates

\begin{abstract}
In this paper, the original $C Q$ algorithm, the relaxed $C Q$ algorithm, the gradient projection method (GPM) algorithm, and the subgradient projection method (SPM) algorithm for the convex split feasibility problem are reviewed, and a renewed $S P M$ algorithm with $S$-subdifferential functions to solve nonconvex split feasibility problems in finite dimensional spaces is suggested. The weak convergence theorem is established.
\end{abstract}

Keywords: S-subgradient projection method; nonconvex; S-subdifferentiable; split feasibility problem

MSC: 47J25; 47H10; 58C20; 49J50; 46T20

\section{Introduction}

The split feasibility problem [1] (subgradient projection method $(S P M)$ ) is the issue of finding a vector $u$ satisfying:

$$
u \in C \text { and } A u \in Q ;
$$

here, both the nonempty underlying sets $C \subseteq \mathbb{R}^{n}$ and $Q \subseteq \mathbb{R}^{m}$ are closed convex, and $A$ is a matrix of $m$ rows and $n$ columns. Since the $S F P$ was raised by Censor [1], it has been rapidly applied in signal processing [2], image restoration [3], intensity modulated radiation therapy (IMRT) [4], and other fields. Besides, different types of iterative algorithms are used to solve the SFP (see [4-22] and the references therein).

The original algorithm used to solve SFP appeared in [1] involved calculating the inverse of matrix $A$ (not necessarily symmetrical, and suppose the inverse $A^{-1}$ exists). In fact, it is very difficult to calculate the inverse of matrix $A$. Thus, the following $C Q$ algorithm presented by Byrne [3] seemed to be more popular:

$$
u_{k+1}=P_{C}\left(u_{k}-\rho_{k} A^{*}\left(I-P_{Q}\right) A u_{k}\right), \quad k \geq 1,
$$

where $P_{C}$ and $P_{Q}$ represent the vertical projections on $C$ and $Q$, respectively, the initial value $u_{1} \in \mathbb{R}^{n}$, $A^{*}$ means the adjoint of $A$, and $\rho_{k} \in(0,2 / \sigma)$ with $\sigma$ relating to the spectral radius of the matrix $A^{*} A$. 
In some other references [2,10], they wrote the spectral radius of the matrix $A^{*} A$ by $\|A\|^{2}$. In the sequel, $\|\cdot\|$ means the two-norm. It is found that Algorithm (1) is a special example of the gradient projection method [10] (GPM) associated with convex minimization. That is, let:

$$
f(u)=\frac{1}{2}\left\|A u-P_{Q}(A u)\right\|^{2},
$$

and consider the convex minimization problem [10]:

$$
\min _{u \in C} f(u)
$$

Recall that the GPM algorithm for the above convex minimization problem is:

$$
u_{k+1}=P_{C}\left(u_{k}-\rho_{k} \nabla f\left(u_{k}\right)\right), \quad k \geq 1,
$$

The stepsize $\rho_{k}$ in the $C Q$ algorithm (1) and the GPM algorithm (2) depends heavily on the matrix norm $\|A\|$. However, it is difficult to calculate or estimate the norm $\|A\|$ in reality. Thus, another way to construct a different stepsize independent of norm $\|A\|$ is expected. Yang [23] proposed the following stepsize:

$$
\rho_{k}=\frac{\lambda_{k}}{\left\|\nabla f\left(x_{k}\right)\right\|}
$$

where $\lambda_{k}$ satisfies:

$$
\sum_{k=1}^{\infty} \lambda_{k}=\infty \text { and } \sum_{k=1}^{\infty} \lambda_{k}^{2}<\infty
$$

Yang [23] proved the convergence of the GPM algorithm (2) under (3) and (4). Besides, the following two more conditions are needed:

- The boundedness of subset $Q$;

- The full column rank of matrix $A$.

However, the conditions above are still very strict, so the application area of the GPM algorithm (2) is limited. Thus, López et al. [2] renewed the stepsize (3) as:

$$
\rho_{k}=\frac{\lambda_{k} f\left(x_{k}\right)}{\left\|\nabla f\left(x_{k}\right)\right\|^{2}}, \quad 0<\lambda_{k}<4 .
$$

Then, López et al. [2] analyzed the weak convergence of the GPM algorithm (2) with the stepsize (5).

On the other hand, although $C$ and $Q$ are convex sets, the projections onto them may not be easy to implement. To overcome this difficulty, Yang [24] presented the relaxed $C Q$ algorithm, in which $C_{0}=\left\{u \in \mathbb{R}^{n}: c(u) \leq 0\right\}$ and $Q_{0}=\left\{v \in \mathbb{R}^{m}: q(v) \leq 0\right\}$ are lower level sets of subdifferentiable convex functions $c: \mathbb{R}^{n} \rightarrow \mathbb{R}$ and $q: \mathbb{R}^{m} \rightarrow \mathbb{R}$ at zero, respectively. Recall that the relaxed $C Q$ algorithm:

$$
u_{k+1}=P_{C_{k, 0}}\left(u_{k}-\rho_{k} A^{*}\left(I-P_{Q_{k, 0}}\right) A u_{k}\right), \quad \rho_{k} \in\left(0,2 /\|A\|^{2}\right), \quad k \geq 1,
$$

where:

$$
C_{k, 0}=\left\{u \in \mathbb{R}^{n}: c\left(u_{k}\right)+\left\langle\phi_{k}, u-u_{k}\right\rangle \leq 0\right\}, \quad \phi_{k} \in \partial c\left(u_{k}\right),
$$

and:

$$
Q_{k, 0}=\left\{v \in \mathbb{R}^{m}: q\left(A u_{k}\right)+\left\langle\varphi_{k}, v-A u_{k}\right\rangle \leq 0\right\}, \quad \varphi_{k} \in \partial q\left(A u_{k}\right) .
$$

Define a function:

$$
f_{k}(u)=\frac{1}{2}\left\|A u-P_{Q_{k, 0}}(A u)\right\|^{2}
$$


hence, its gradient:

$$
\nabla f_{k}(u)=A^{*}\left(A u-P_{Q_{k, 0}}(A u)\right) .
$$

López et al. [2] improved this relaxed CQ algorithm (6) as follows:

$$
u_{k+1}=P_{C_{k, 0}}\left(u_{k}-\rho_{k} \nabla f_{k}\left(u_{k}\right)\right), \quad k \geq 1,
$$

where:

$$
\rho_{k}=\frac{\lambda_{k} f_{k}\left(u_{k}\right)}{\left\|\nabla f_{k}\left(u_{k}\right)\right\|^{2}}, \quad 0<\lambda_{k}<4 .
$$

Thus, the convergence of Algorithm (8) with the stepsize (9) need not calculate or estimate the norm of matrix $A$.

Guo [25] reformulated the relaxed CQ algorithm (6) into a subgradient projection method (SPM) by studying the subgradient projector of convex continuous functions. He denoted the subgradient projector related to $\left(c\right.$, zero, $\left.s_{c}\right)$ and $\left(f_{k}\right.$, zero, $\left.\nabla f_{k}\right)$ by $G_{c^{0}}$ and $G_{f_{k}^{0}}$, respectively. Let $R_{\lambda_{k}, f_{k}^{0}}=I+$ $\lambda_{k}\left(G_{f_{k}^{0}}-I\right)$, then:

$$
u_{k+1}=G_{c^{0}}\left(R_{\lambda_{k}, f_{k}^{0}}\left(u_{k}\right)\right), \quad 0<\lambda_{k}<2,
$$

converges iteratively to a point $\widetilde{u}$ such that $\widetilde{u} \in C_{0}$ and $A \widetilde{u} \in Q_{0}$.

In this paper, the $C Q$ algorithm (1), the relaxed $C Q$ algorithm (6), the GPM algorithm (2), and the SPM algorithm (10) for the convex SFP are reviewed, the definition of the $S$-subdifferential with respect to a set $S$ is introduced, the $S F P$ is generalized to a nonconvex case where the functions $c$ and $q$ are both continuous and $S$-subdifferentiable, then the supposed algorithm converges iteratively to a solution of nonconvex SFP. The $S$-subgradient projector of a continuous function has a pivotal role in structuring the iterative algorithm to solve the nonconvex SFP.

\section{Preliminaries}

First of all, we write $u_{k} \rightarrow u$ [5] to show that $\left\{u_{k}\right\}$ converges weakly to $u$. Let nonempty set $S \subseteq \mathbb{R}^{n}$ be closed and the vertical projection [16] $P_{S}$ from $\mathbb{R}^{n}$ onto $S$ be defined by the following form:

$$
P_{S}(u):=\operatorname{argmin}_{v \in S}\|u-v\|, \quad \forall u \in \mathbb{R}^{n} .
$$

Definition 1. [26] Let $f: \mathbb{R}^{n} \rightarrow(-\infty,+\infty)$.

The domain of $f$ is:

$$
\operatorname{dom} f=\left\{u \in \mathbb{R}^{n}: f(u)<+\infty\right\} .
$$

The graph of $f$ is:

$$
\text { graf }=\left\{(u, \xi) \in \mathbb{R}^{n} \times \mathbb{R}: f(u)=\xi\right\} .
$$

The epigraph of $f$ is:

$$
\text { epif }=\left\{(u, \xi) \in \mathbb{R}^{n} \times \mathbb{R}: f(u) \leq \xi\right\} .
$$

The lower level set of $f$ at height $\xi \in \mathbb{R}$ is:

$$
l e v_{\leq \xi} f=\left\{u \in \mathbb{R}^{n}: f(u) \leq \xi\right\} .
$$

To define S-subgradient projector of continuous functions, we need the following definition.

Definition 2 ([25]). Given a set $S \subseteq \mathbb{R}^{n}$ and a constant $r_{f}>0$, a vector $x \in \mathbb{R}^{n}$ is said to be an $S$-subgradient of function $f: \mathbb{R}^{n} \rightarrow \mathbb{R}$ at $u$ if:

$$
\langle v-u, x\rangle+f(u)+\frac{r_{f}}{2} d_{S}^{2}(u) \leq f(v)+\frac{r_{f}}{2} d_{S}^{2}(v), \quad \forall v \in \mathbb{R}^{n} .
$$


The set of all S-subgradients of function $f$ at $u$ is called the S-subdifferential of $f$ at $u$ and is denoted by:

$$
\partial_{S, r_{f}} f(u)=\left\{x \in \mathbb{R}^{n}:\langle v-u, x\rangle+f(u)+\frac{r_{f}}{2} d_{S}^{2}(u) \leq f(v)+\frac{r_{f}}{2} d_{S}^{2}(v), \quad \forall v \in \mathbb{R}^{n}\right\}
$$

where $d_{S}(u)=\inf _{v \in S}\|u-v\|$ is the usual distance related to the two-norm from point $u$ to set $S$.

Note that if $S=\mathbb{R}^{n}$, the $S$-subdifferential collapses to the Fenchel subdifferential; so does $r=0$. The definition of the Fenchel subdifferential is given below.

Definition 3 ([26]). Let $f: \mathbb{R}^{n} \rightarrow(-\infty,+\infty)$ (not necessarily convex), and define its Fenchel subdifferential at $u$,

$$
\partial f(u):=\left\{x \in \mathbb{R}^{n}:\langle v-u, x\rangle+f(u) \leq f(v), \quad \forall v \in \mathbb{R}^{n}\right\} .
$$

When $f$ is convex, $\partial f(u)$ is the usual subdifferential.

Lemma 1 ([25]). Let $C_{\xi}=l e v_{\leq \xi} f \neq \varnothing, C_{\xi} \subseteq S \subseteq \mathbb{R}^{n}, S$ be closed and convex, and $f: \mathbb{R}^{n} \rightarrow \mathbb{R}$ be the $S$-subdifferential on $\mathbb{R}^{n}$. Then, there exists a constant $r_{f}>0$ and for any $u \notin C_{\xi}$ such that:

$$
s_{f}(u) \in \partial_{s, r_{f}} f(u) \Rightarrow s_{f}(u) \neq 0 .
$$

Therefore, we can define the $S$-subgradient projector.

Definition 4 ([25]). Assume that $f: \mathbb{R}^{n} \rightarrow \mathbb{R}$ is continuous and $S$-subdifferential on $\mathbb{R}^{n}$ with respect to $S$. Let the lower level sets of $f$ at height $\xi \in \mathbb{R}$ be such that $C_{\xi}=l e v_{\leq \xi} f \neq \varnothing$. Let $C_{\xi} \subseteq S \subseteq \mathbb{R}^{n}$ and $S$ be closed and convex. Assume that $\partial_{S, r_{f}} f(u)$ is the $S$-subdifferential of $f$ with respect to $S$ and $s_{f}(u) \in \partial_{S, r_{f}} f(u)$. The $S$-subgradient projector onto $C_{\xi}$ related to $\left(f, \xi, s_{f}\right)$ is:

$$
\begin{aligned}
& G_{S, f \tilde{\xi}}: \mathbb{R}^{n} \rightarrow \mathbb{R}^{n} \\
& u \mapsto \begin{cases}u+\frac{\tilde{\xi}-f(u)}{\left\|s_{f}(u)\right\|^{2}} s_{f}(u), & u \notin C_{\tilde{\xi}} \\
u, & u \in C_{\tilde{\xi}} .\end{cases}
\end{aligned}
$$

Lemma 2 ([25]). Let $S \subseteq \mathbb{R}^{n}$ be closed and convex and $f: \mathbb{R}^{n} \rightarrow \mathbb{R}$ be the $S$-subdifferential on $\mathbb{R}^{n}$. Then, there exists a constant $r_{f}>0$ such that:

$$
x \in \partial_{S, r_{f}} f(u) \Leftrightarrow x \in \partial f(u)+r_{f}\left(I-P_{S}\right)(u) .
$$

\section{Nonconvex Split Feasibility Problem}

In this part, we take a look at the nonconvex split feasibility problem. Let us look at some hypothetical situations. Assume that:

(1) continuous, but not necessarily convex functions $c: \mathbb{R}^{n} \rightarrow \mathbb{R}$ and $q: \mathbb{R}^{m} \rightarrow \mathbb{R}$ are the $S$-subdifferential, and $c$ and $q$ are locally Lipschitzian in addition.

(2) the lower level sets of $c$ and $q$ at height $\xi \in \mathbb{R}, \xi>0$ are defined by $C_{\xi}=\left\{u \in \mathbb{R}^{n}: c(u) \leq \xi\right\}$ and $Q_{\xi}=\left\{v \in \mathbb{R}^{m}: q(v) \leq \xi\right\}$.

(3) the set of solutions to SFP is nonempty, that is there exists at least one element $\widetilde{u} \in C_{\xi}$ such that $A \widetilde{u} \in Q_{\tilde{\xi}}$, where $A$ is an $m \times n$ matrix.

(4) $U \subseteq \mathbb{R}^{n}$ and $V \subseteq \mathbb{R}^{m}$ are closed convex subsets such that $C_{\xi} \subseteq U$ and $Q_{\xi} \subseteq V$.

(5) $\quad c$ and $q$ are the $S$-subdifferential on $\mathbb{R}^{n}$ and $\mathbb{R}^{m}$ with respect to $U$ and $V$, respectively.

(6) $\partial_{U, r_{c}} c(u)$ and $\partial_{V, r_{q}} q(v)$ are the $S$-subdifferential of $c$ and $q$ with respect to $U$ and $V$, respectively.

(7) both $\partial_{U, r_{c}} c(u)$ and $\partial_{V, r_{q}} q(v)$ are not empty; let $s_{c}(u) \in \partial_{U, r_{c}} c(u)$ and $s_{q}(v) \in \partial_{V, r_{q}} q(v)$. 
In such conditions, the $S$-subgradient projector onto $C_{\xi}$ related to $\left(c, \xi, s_{\mathcal{c}}\right)$ is:

$$
\begin{aligned}
& G_{U, c^{\xi}}: \mathbb{R}^{n} \rightarrow \mathbb{R}^{n} \\
& u \mapsto \begin{cases}u+\frac{\xi-c(u)}{\left\|s_{c}(u)\right\|^{2}} s_{\mathcal{C}}(u), & u \notin C_{\tilde{\zeta}} \\
u, & u \in C_{\xi} .\end{cases}
\end{aligned}
$$

The $S$-subgradient projector onto $Q_{\xi}$ related to $\left(q, \xi, s_{q}\right)$ is:

$$
\begin{aligned}
& G_{V, q^{\xi}}: \mathbb{R}^{m} \rightarrow \mathbb{R}^{m} \\
& v \mapsto \begin{cases}v+\frac{\xi-q(v)}{\left\|s_{q}(v)\right\|^{2}} s_{q}(v), & v \notin Q_{\xi} \\
v, & v \in Q_{\xi} .\end{cases}
\end{aligned}
$$

For $k \geq 1$ and $\phi_{k} \in \partial_{u, r_{c}} c\left(u_{k}\right)$, give a set:

$$
C_{k, \xi}=\left\{u \in \mathbb{R}^{n}: c\left(u_{k}\right)+\left\langle\phi_{k}, u-u_{k}\right\rangle \leq \xi\right\},
$$

and for $\varphi_{k} \in \partial_{V, r_{q}} q\left(A u_{k}\right)$, give another set:

$$
Q_{k, \xi}=\left\{v \in \mathbb{R}^{m}: q\left(A u_{k}\right)+\left\langle\varphi_{k}, v-A u_{k}\right\rangle \leq \xi\right\} .
$$

Then, we can define a function like (7),

$$
f_{k}(u)=\frac{1}{2}\left\|A u-P_{Q_{k, \tilde{\xi}}}(A u)\right\|^{2},
$$

where the set $Q_{k, \xi}$ is mentioned in (13), so the gradient of $f_{k}$ at $u$ is:

$$
\nabla f_{k}(u)=A^{*}\left(A u-P_{Q_{k, \xi}}(A u)\right) .
$$

Then, we can improve the relaxed $C Q$ algorithm by:

$$
u_{k+1}=P_{C_{k, \tilde{\xi}}}\left(u_{k}-\rho_{k} \nabla f_{k}\left(u_{k}\right)\right)
$$

where:

$$
\rho_{k}=\frac{\lambda_{k} f_{k}\left(u_{k}\right)}{\left\|\nabla f_{k}\left(u_{k}\right)\right\|^{2}} .
$$

For any $u_{k} \in \mathbb{R}^{n}$, by [27], we get:

$$
\begin{aligned}
P_{C_{k, \xi}}\left(u_{k}\right) & =u_{k}+\frac{\left(\xi-c\left(u_{k}\right)+\left\langle\phi_{k}, u_{k}\right\rangle\right)-\left\langle\phi_{k}, u_{k}\right\rangle}{\left\|\phi_{k}\right\|^{2}} \phi_{k} \\
& =u_{k}+\frac{\xi-c\left(u_{k}\right)}{\left\|\phi_{k}\right\|^{2}} \phi_{k} \\
& =G_{U, c \tilde{\xi}}\left(u_{k}\right) .
\end{aligned}
$$

Denote the $S$-subgradient projector related to $\left(f_{k}, 0, \nabla f_{k}\right)$ by $G_{f_{k}^{0}}$, that is,

$$
\begin{aligned}
& G_{f_{k}^{0}}: \mathbb{R}^{n} \rightarrow \mathbb{R}^{n} \\
& u \mapsto \begin{cases}u+\frac{-f_{k}(u)}{\left\|\nabla f_{k}(u)\right\|^{2}} \nabla f_{k}(u), & A u \notin Q_{k, \xi} \\
u, & A u \in Q_{k, \zeta}\end{cases}
\end{aligned}
$$


Let $R_{\lambda_{k}, f_{k}^{0}}=I+\lambda_{k}\left(G_{f_{k}^{0}}-I\right)$, and by (14), we obtain:

$$
\begin{aligned}
u_{k+1} & =P_{C_{k, \xi}}\left(u_{k}-\rho_{k} \nabla f_{k}\left(u_{k}\right)\right) \\
& =G_{U, c^{\xi}}\left(u_{k}-\frac{\lambda_{k} f_{k}\left(u_{k}\right)}{\left\|\nabla f_{k}\left(u_{k}\right)\right\|^{2}} \nabla f_{k}\left(u_{k}\right)\right) \\
& =G_{U, c^{\xi}}\left(R_{\lambda_{k}, f_{k}^{0}}\left(u_{k}\right)\right) .
\end{aligned}
$$

Now, we suggest the $S$-subgradient projection method with the $S$-subdifferential functions for solving nonconvex SFP by:

$$
u_{k+1}=G_{U, c^{\xi}}\left(R_{\lambda_{k}, f_{k}^{0}}\left(u_{k}\right)\right) .
$$

Theorem 1. Assume that (1)-(7) are satisfied and $\inf _{k} \lambda_{k}\left(2-\lambda_{k}\right)>0$. Then, $\left\{u_{n}\right\}$ generated by (16) weakly converges to a point $\widetilde{u}$ such that $\widetilde{u} \in C_{\xi}$ and $A \widetilde{u} \in Q_{\xi}$.

Proof. Let $w$ be any point in the solution set; that is, $w \in C_{\xi}$ and $A w \in Q_{\xi}$. Since $\varphi_{k} \in \partial_{V, r_{q}} q\left(A u_{k}\right)$, for any $A w \in Q_{\xi}$, from (11), we attain:

$$
\begin{aligned}
q\left(A u_{k}\right)+\left\langle\varphi_{k}, A w-A u_{k}\right\rangle & \leq q(A w)+\frac{r_{q}}{2} d_{V}^{2}(A w)-\frac{r_{q}}{2} d_{V}^{2}\left(A u_{k}\right) \\
& =q(A w)-\frac{r_{q}}{2} d_{V}^{2}\left(A u_{k}\right) \\
& \leq q(A w) \leq \xi .
\end{aligned}
$$

Hence, we achieve $A w \in Q_{k, \xi}$. Moreover, $f_{k}(w)=0$.

Next, we consider two cases.

If $A u_{k} \in Q_{k, \tilde{\zeta}}$, by the definition of $G_{f_{k}^{0}}$, then:

$$
\left\langle G_{f_{k}^{0}}\left(u_{k}\right)-w, G_{f_{k}^{0}}\left(u_{k}\right)-u_{k}\right\rangle=\left\langle G_{f_{k}^{0}}\left(u_{k}\right)-w, u_{k}-u_{k}\right\rangle=0 .
$$

If $A u_{k} \notin Q_{k, \xi}$, it is deduced from (12), (15) and $f_{k}(w)=0$ that:

$$
\begin{aligned}
\left\langle G_{f_{k}^{0}}\left(u_{k}\right)-w, G_{f_{k}^{0}}\left(u_{k}\right)-u_{k}\right\rangle & =\left\langle u_{k}+\frac{-f_{k}\left(u_{k}\right)}{\left\|\nabla f_{k}\left(u_{k}\right)\right\|^{2}} \nabla f_{k}\left(u_{k}\right)-w, u_{k}+\frac{-f_{k}\left(u_{k}\right)}{\left\|\nabla f_{k}\left(u_{k}\right)\right\|^{2}} \nabla f_{k}\left(u_{k}\right)-u_{k}\right\rangle \\
& =\left\langle u_{k}-w, \frac{-f_{k}\left(u_{k}\right)}{\left\|\nabla f_{k}\left(u_{k}\right)\right\|^{2}} \nabla f_{k}\left(u_{k}\right)\right\rangle+\frac{f_{k}^{2}\left(u_{k}\right)}{\left\|\nabla f_{k}\left(u_{k}\right)\right\|^{2}} \\
& =\frac{f_{k}\left(u_{k}\right)}{\left\|\nabla f_{k}\left(u_{k}\right)\right\|^{2}}\left\langle w-u_{k}, \nabla f_{k}\left(u_{k}\right)\right\rangle+\frac{f_{k}^{2}\left(u_{k}\right)}{\left\|\nabla f_{k}\left(u_{k}\right)\right\|^{2}} \\
& \leq \frac{f_{k}\left(u_{k}\right)}{\left\|\nabla f_{k}\left(u_{k}\right)\right\|^{2}}\left(f_{k}(w)-f_{k}\left(u_{k}\right)\right)+\frac{f_{k}^{2}\left(u_{k}\right)}{\left\|\nabla f_{k}\left(u_{k}\right)\right\|^{2}} \\
& =0 .
\end{aligned}
$$

Whether or not $A u_{k}$ belongs to $Q_{k, \xi}$, we have:

$$
\left\langle G_{f_{k}^{0}}\left(u_{k}\right)-w, G_{f_{k}^{0}}\left(u_{k}\right)-u_{k}\right\rangle \leq 0 .
$$

Likewise, we get:

$$
\left\langle G_{U, c^{\tilde{\zeta}}}\left(R_{\lambda_{k}, f_{k}^{0}}\left(u_{k}\right)\right)-w, G_{U, c \tilde{\zeta}}\left(R_{\lambda_{k}, f_{k}^{0}}\left(u_{k}\right)\right)-R_{\lambda_{k}, f_{k}^{0}}\left(u_{k}\right)\right\rangle \leq 0 .
$$


From the definition of $R_{\lambda_{k}, f_{k}^{0}}$ and (17), we estimate:

$$
\begin{aligned}
\left\|R_{\lambda_{k}, f_{k}^{0}}\left(u_{k}\right)-w\right\|^{2}= & \left\|u_{k}+\lambda_{k}\left(G_{f_{k}^{0}}\left(u_{k}\right)-u_{k}\right)-w\right\|^{2} \\
= & \left\|u_{k}-w\right\|^{2}+2 \lambda_{k}\left\langle u_{k}-G_{f_{k}^{0}}\left(u_{k}\right), G_{f_{k}^{0}}\left(u_{k}\right)-u_{k}\right\rangle \\
& +2 \lambda_{k}\left\langle G_{f_{k}}\left(u_{k}\right)-w, G_{f_{k}^{0}}\left(u_{k}\right)-u_{k}\right\rangle+\lambda_{k}^{2}\left\|G_{f_{k}^{0}}\left(u_{k}\right)-u_{k}\right\|^{2} \\
\leq & \left\|u_{k}-w\right\|^{2}-\lambda_{k}\left(2-\lambda_{k}\right)\left\|G_{f_{k}^{0}}\left(u_{k}\right)-u_{k}\right\|^{2} .
\end{aligned}
$$

This together with (16) and (18) implies that:

$$
\begin{aligned}
\left\|u_{k+1}-w\right\|^{2} & =\left\|G_{U, c^{\xi}}\left(R_{\lambda_{k}, f_{k}^{0}}\left(u_{k}\right)\right)-w\right\|^{2} \\
& =\left\|R_{\lambda_{k}, f_{k}^{0}}\left(u_{k}\right)+G_{U, c^{\xi}}\left(R_{\lambda_{k}, f_{k}^{0}}\left(u_{k}\right)\right)-R_{\lambda_{k}, f_{k}^{0}}\left(u_{k}\right)-w\right\|^{2} \\
& \leq\left\|R_{\lambda_{k}, f_{k}^{0}}\left(u_{k}\right)-w\right\|^{2}-\left\|G_{U, c^{\xi}}\left(R_{\lambda_{k}, f_{k}^{0}}\left(u_{k}\right)\right)-R_{\lambda_{k}, f_{k}^{0}}\left(u_{k}\right)\right\|^{2} \\
& \leq\left\|u_{k}-w\right\|^{2}-\lambda_{k}\left(2-\lambda_{k}\right)\left\|G_{f_{k}^{0}}\left(u_{k}\right)-u_{k}\right\|^{2}-\left\|G_{U, c^{\xi}}\left(R_{\lambda_{k}, f_{k}^{0}}\left(u_{k}\right)\right)-R_{\lambda_{k}, f_{k}^{0}}\left(u_{k}\right)\right\|^{2} .
\end{aligned}
$$

By $\inf _{k} \lambda_{k}\left(2-\lambda_{k}\right)>0$, we gain the Fejér monotonicity:

$$
\left\|u_{k+1}-w\right\|^{2} \leq\left\|u_{k}-w\right\|^{2}, \quad k \geq 1 .
$$

Thus, we receive the existence of $\lim _{k \rightarrow \infty}\left\|u_{k}-w\right\|$, so the boundedness of $\left\{u_{k}\right\}$ is obtained.

By (19), we can find:

$$
\lambda_{k}\left(2-\lambda_{k}\right)\left\|G_{f_{k}^{0}}\left(u_{k}\right)-u_{k}\right\|^{2}+\left\|G_{U, c^{\tilde{\xi}}}\left(R_{\lambda_{k}, f_{k}^{0}}\left(u_{k}\right)\right)-R_{\lambda_{k}, f_{k}^{0}}\left(u_{k}\right)\right\|^{2} \leq\left\|u_{k}-w\right\|^{2}-\left\|u_{k+1}-w\right\|^{2} .
$$

By $\inf _{k} \lambda_{k}\left(2-\lambda_{k}\right)>0$ and let $v_{k}=R_{\lambda_{k}, f_{k}^{0}}\left(u_{k}\right)$, we get:

$$
\lim _{k \rightarrow \infty}\left\|G_{f_{k}^{0}}\left(u_{k}\right)-u_{k}\right\|=\lim _{k \rightarrow \infty}\left\|G_{U, c^{\xi}}\left(v_{k}\right)-v_{k}\right\|=0
$$

One can see that:

$$
\left\|G_{f_{k}^{0}}\left(u_{k}\right)-u_{k}\right\|=\left\|u_{k}+\frac{-f_{k}\left(u_{k}\right)}{\left\|\nabla f_{k}\left(u_{k}\right)\right\|^{2}} \nabla f_{k}\left(u_{k}\right)-u_{k}\right\|=\frac{f_{k}\left(u_{k}\right)}{\left\|\nabla f_{k}\left(u_{k}\right)\right\|} .
$$

We observe from $\nabla f_{k}(w)=0$ that:

$$
\left\|\nabla f_{k}\left(u_{k}\right)\right\|=\left\|\nabla f_{k}\left(u_{k}\right)-\nabla f_{k}(w)\right\| \leq\|A\|^{2}\left\|u_{k}-w\right\| .
$$

Therefore, $\left\{\nabla f_{k}\left(u_{k}\right)\right\}$ is bounded. From (20) and (21), we have $\lim _{k \rightarrow \infty} f_{k}\left(u_{k}\right)=0$, which means:

$$
\lim _{k \rightarrow \infty}\left\|A u_{k}-P_{Q_{k, \tilde{\xi}}} A u_{k}\right\|=0
$$

Since $q$ is locally Lipschitz, we have the local boundedness of $\partial q$; therefore, we get that $\partial q$ is bounded on the bounded set; so is $I-P_{S}$. From Lemma 2, we obtain that $\partial_{V, r_{q}} q$ is bounded on the bounded set; thus, there exists $\delta>0$ such that $\left\|\varphi_{k}\right\| \leq \delta$. Since $P_{Q_{k, \xi}} A u_{k} \in Q_{k, \tilde{\xi}}$, we conclude:

$$
q\left(A u_{k}\right) \leq \xi+\left\langle\varphi_{k}, A u_{k}-P_{Q_{k, \xi}}\left(A u_{k}\right)\right\rangle \leq \xi+\delta\left\|A u_{k}-P_{Q_{k, \xi}}\left(A u_{k}\right)\right\| .
$$


As $\left\{u_{k}\right\}$ is bounded, we can find a subsequence $\left\{u_{k_{i}}\right\}$ of $\left\{u_{k}\right\}$ such that $u_{k_{i}} \rightarrow \widetilde{u}$. Then, the continuity of $q$ and (22) imply that:

$$
q(A \widetilde{u})=\lim _{i \rightarrow \infty} q\left(A u_{k_{i}}\right) \leq \xi .
$$

Hence, $A \widetilde{u} \in Q_{\tilde{\zeta}}$.

Since $v_{k}=R_{\lambda_{k}, f_{k}^{0}}\left(u_{k}\right)$, we have $v_{k_{i}}=R_{\lambda_{k_{i}} f_{k_{i}}^{0}}\left(u_{k_{i}}\right)$, and then, from (20), we have that:

$$
\lim _{i \rightarrow \infty}\left\|v_{k_{i}}-u_{k_{i}}\right\|=\lim _{i \rightarrow \infty} \lambda_{k_{i}}\left\|G_{f_{k_{i}}^{0}}\left(u_{k_{i}}\right)-u_{k_{i}}\right\|=0 .
$$

Since $u_{k_{i}} \rightarrow \widetilde{u}$, we have $v_{k_{i}} \rightarrow \widetilde{u}$. Next, two cases are considered.

If $v_{k_{i}} \in C_{\tilde{\xi}}$, i.e., $c\left(v_{k_{i}}\right) \leq \xi$ and $G_{U, c \xi}\left(v_{k_{i}}\right)=v_{k_{i}}$, so

$$
\max \left\{c\left(v_{k_{i}}\right)-\xi, 0\right\}=0
$$

and:

$$
\left\|s_{\mathcal{C}}\left(v_{k_{i}}\right)\right\|\left\|G_{U, c^{\tilde{\xi}}}\left(v_{k_{i}}\right)-v_{k_{i}}\right\|=0 .
$$

Hence, $\max \left\{c\left(v_{k_{i}}\right)-\xi, 0\right\}=\left\|s_{c}\left(v_{k_{i}}\right)\right\|\left\|G_{U, c \xi}\left(v_{k_{i}}\right)-v_{k_{i}}\right\|$.

If $v_{k_{i}} \notin C_{\tilde{\xi}}$, i.e., $c\left(v_{k_{i}}\right)>\xi$, hence, $\max \left\{c\left(v_{k_{i}}\right)-\xi, 0\right\}=c\left(v_{k_{i}}\right)-\xi$.

$$
\left\|s_{\mathcal{c}}\left(v_{k_{i}}\right)\right\|\left\|G_{U, c^{\xi}}\left(v_{k_{i}}\right)-v_{k_{i}}\right\|=\left\|s_{c}\left(v_{k_{i}}\right)\right\|\left\|v_{k_{i}}+\frac{\xi-c\left(v_{k_{i}}\right)}{\left\|s_{\mathcal{c}}\left(v_{k_{i}}\right)\right\|^{2}} s_{c}\left(v_{k_{i}}\right)-v_{k_{i}}\right\|=c\left(v_{k_{i}}\right)-\xi .
$$

No matter whether $v_{k_{i}}$ belongs to $C_{\xi}$ or not, we have $\max \left\{c\left(v_{k_{i}}\right)-\xi, 0\right\}=\left\|s_{c}\left(v_{k_{i}}\right)\right\|\left\|G_{U, \tilde{c} \xi}\left(v_{k_{i}}\right)-v_{k_{i}}\right\|$.

From Lemma 2, there exists $\kappa>0$ such that $\left\{v_{k_{i}}\right\}$ lies in $B(\widetilde{u} ; \kappa)$ and:

$$
\tau=\sup \|\partial c(B(\tilde{x} ; \kappa))\|+r_{c} \sup _{i \geq 1}\left\|\left(I-P_{S}\right) v_{k_{i}}\right\|<+\infty .
$$

Hence,

$$
\left\|s_{\mathcal{c}}\left(v_{k_{i}}\right)\right\| \leq \tau, \quad i \geq 1
$$

By (20), we have:

$$
\max \{c(\widetilde{u})-\xi, 0\} \leq \lim _{i \rightarrow \infty} \max \left\{c\left(v_{k_{i}}\right)-\xi, 0\right\} \leq \tau \lim _{i \rightarrow \infty}\left\|G_{U, c \tilde{\xi}}\left(v_{k_{i}}\right)-v_{k_{i}}\right\|=0 .
$$

Thus, $c(\widetilde{u}) \leq \xi$, in other words, $\widetilde{u} \in C_{\tilde{\xi}}$; this together with $A \widetilde{u} \in Q_{\xi}$ shows that the proof is done.

Remark 1. We raise two questions:

1. Can the result presented in Theorem 1 hold in infinity spaces?

2, Since we only obtain weak convergence of the proposed algorithm in this paper, how do we modify the algorithm so that the strong convergence is guaranteed?

Remark 2. Let $\left\{\lambda_{k}\right\}$ be a sequence such that $\inf _{k} \lambda_{k}\left(2-\lambda_{k}\right)>0$, but in the process of proving the convergence of the subgradient projection algorithm, Guo [25] used $\lambda_{k}=1$ in particular. In our proof, we do not use that.

\section{Conclusions}

In this paper, we studied the SFP in the nonconvex case. In finite dimensional spaces, we gave two $S$-subdifferentiable functions and then structured nonconvex sets based on the epigraph. By the nonzero of the $S$-subgradient of the $S$-subdifferentiable function, we introduced the $S$-subgradient 
projector of the continuous function, but not necessarily convex. Under this $S$-subgradient projector, we transferred the GPM into the SPM, that is we suggested the $S$-subgradient projection method with $S$-subdifferential functions for solving nonconvex SFP. The weak convergence theorem was guaranteed.

Author Contributions: All authors participated in the conceptualization, validation, formal analysis, and investigation, as well as the writing of the original draft preparation, reviewing, and editing.

Funding: This work was supported by the Key Subject Program of Lingnan Normal University (1171518004), the Natural Science Foundation of Guangdong Province (2018A0303070012), the Young Innovative Talents Project at Guangdong Universities (2017KQNCX125), and the Ph.D. research startup foundation of Lingnan Normal University (ZL1919). Yonghong Yao was supported in part by the grant TD13-5033.

Conflicts of Interest: The authors declare that they have no competing interests.

\section{References}

1. Censor, Y.; Elfving, T. A multiprojection algorithm using Bregman projections in a product space. Numer. Algorithms 1994, 8, 221-239. [CrossRef]

2. López, G.; Martín, V.; Wang, F.; Xu, H. Solving the split feasibility problem without prior knowledge of matrix norms. Inverse Probl. 2012, 28, 085004. [CrossRef]

3. Byrne, C. Iterative oblique projection onto convex subsets and the split feasibility problem. Inverse Probl. 2002, 18, 441-453. [CrossRef]

4. Censor, Y.; Motova, A.; Segal, A. Perturbed projections and subgradient projections for the multiple-sets split feasibility problem. J. Math. Anal. Appl. 2007, 327, 1244-1256. [CrossRef]

5. Ceng, L.; Petruşel, A.; Yao, J. Relaxed extragradient methods with regularization for general system of variational inequalities with constraints of split feasibility and fixed point problems. In Abstract and Applied Analysis; Hindawi: London, UK, 2013.

6. Ceng, L.; Wong, M.; Petruşel, A.; Yao, J. Relaxed implicit extragradient-like methods for finding minimum-norm solutions of the split feasibility problem. Fixed Point Theory 2013, 14, 327-344.

7. Ceng, L.C.; Petrusel, A.; Yao, J.C.; Yao, Y. Hybrid viscosity extragradient method for systems of variational inequalities, fixed Points of nonexpansive mappings, zero points of accretive operators in Banach spaces. Fixed Point Theory 2018, 19, 487-502. [CrossRef]

8. Ceng, L.; Petruşel, A.; Yao, J.; Yao, Y. Systems of variational inequalities with hierarchical variational inequality constraints for Lipschitzian pseudocontractions. Fixed Point Theory 2019, 20, 113-134. [CrossRef]

9. Wang, F; $\mathrm{Xu}, \mathrm{H}$. Approximating curve and strong convergence of the $\mathrm{CQ}$ algorithm for the split feasibility problem. J. Inequal. Appl. 2010, 102085. [CrossRef]

10. $\mathrm{Xu}, \mathrm{H}$. A variable Krasnosel'skii-Mann algorithm and the multiple-set split feasibility problem. Inverse Probl. 2006, 22, 2021-2034. [CrossRef]

11. Chen, J.; Ceng, L.; Qiu, Y.; Kong, Z. Extra-gradient methods for solving split feasibility and fixed point problems. Fixed Point Theory Appl. 2015, 192. [CrossRef]

12. Yao, Y.; Liou, Y.C.; Yao, J.C. Split common fixed point problem for two quasi-pseudocontractive operators and its algorithm construction. Fixed Point Theory Appl. 2015, 2015, 127. [CrossRef]

13. Yao, Y.; Yao, J.; Liou, Y.; Postolache, M. Iterative algorithms for split common fixed points of demicontractive operators without priori knowledge of operator norms. Carpathian J. Math. 2018, 34, 459-466.

14. Yao, Y.; Liou, Y.; Postolache, M. Self-adaptive algorithms for the split problem of the demicontractive operators. Optimization 2018, 67, 1309-1319. [CrossRef]

15. Yao, Y.; Leng, L.; Postolache, M.; Zheng, X. Mann-type iteration method for solving the split common fixed point problem. J. Nonlinear Convex Anal. 2017, 18, 875-882.

16. Yao, Y.; Postolache, M.; Yao, J. An iterative algorithm for solving the generalized variational inequalities and fixed points problems. Mathematics 2019, 7, 61. [CrossRef]

17. Yao, Y.; Liou, Y.; Yao, J. Iterative algorithms for the split variational inequality and fixed point problems under nonlinear transformations. J. Nonlinear Sci. Appl. 2017, 10, 843-854. [CrossRef]

18. Zhang, C.; Zhu, Z.; Yao, Y.; Liu, Q. Homotopy method for solving mathematical programs with bounded box-constrained variational inequalities. Optimization 2019, 68, 2293-2312. [CrossRef] 
19. Zhao, X.P.; Yao, J.C.; Yao, Y. A proximal algorithm for solving split monotone variational inclusions. UPB Sci. Bull. Ser. A 2020, in press.

20. Yao, Y.; Postolache, M.; Liou, Y.C. Strong convergence of a self-adaptive method for the split feasibility problem. Fixed Point Theory Appl. 2013, 201. [CrossRef]

21. Dadashi, V.; Postolache, M. Forward-backward splitting algorithm for fixed point problems and zeros of the sum of monotone operators. Arab. J. Math. 2019. [CrossRef]

22. Yao, Y.; Postolache, M.; Zhu, Z. Gradient methods with selection technique for the multiple-sets split feasibility problem. Optimization 2019. [CrossRef]

23. Yang, Q. On variable-step relaxed projection algorithm for variational inequalities. J. Math. Anal. Appl. 2005, 302, 166-179. [CrossRef]

24. Yang, Q. The relaxed CQ algorithm solving the split feasibility problem. Inverse Probl. 2004, 20, 1261-1266. [CrossRef]

25. Guo, Y. CQ Algorithms: Theory, Computations and Nonconvex Extensions. Master's Thesis, University of British Columbia, Vancouver, BC, Canada, 2014.

26. Bauschke, H.; Combettes, P. Convex Analysis and Monotone Operator Theory in Hilbert Spaces; Springer: New York, NY, USA, 2011.

27. Cegielski, A. Iterative Methods for Fixed Point Problems in Hilbert Spaces; Lecture Notes in Mathematics; Springer: Heidelberg, Germany, 2012.

(c) 2019 by the authors. Licensee MDPI, Basel, Switzerland. This article is an open access article distributed under the terms and conditions of the Creative Commons Attribution (CC BY) license (http://creativecommons.org/licenses/by/4.0/). 